\title{
CMIsTARK: Python package for the Stark-effect calculation and symmetry classification of linear, symmetric and asymmetric top wavefunctions in dc electric fields
}

\author{
Yuan-Pin Chang ${ }^{\mathrm{a}}$, Frank Filsinger ${ }^{\mathrm{b}, 1}$, Boris G. Sartakov ${ }^{\mathrm{c}}$, Jochen Küpper ${ }^{\mathrm{a}, \mathrm{d}, \mathrm{e}, *}$ \\ ${ }^{a}$ Center for Free-Electron Laser Science, DESY, Notkestrasse 85, 22607 Hamburg, Germany \\ ${ }^{\mathrm{b}}$ Fritz-Haber-Institut der MPG, Faradayweg 4-6, 14195 Berlin, Germany

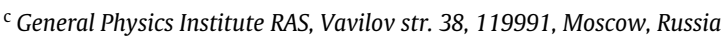 \\ ${ }^{d}$ Department of Physics, University of Hamburg, Luruper Chausse 149, 22761 Hamburg, Germany \\ ${ }^{\mathrm{e}}$ The Hamburg Center for Ultrafast Imaging, Luruper Chaussee 149, 22761 Hamburg, Germany
}

\section{A R T I C L E I N F O}

\section{Article history:}

Received 28 May 2013

Received in revised form

2 September 2013

Accepted 4 September 2013

Available online 13 September 2013

\section{Keywords:}

Molecular rotation

Linear top molecule

Symmetric top molecule

Asymmetric top molecule

Electric field

Stark effect

\begin{abstract}
A B S T R A C T
The Controlled Molecule Imaging group (CMI) at the Center for Free Electron Laser Science (CFEL) has developed the CMISTARK software to calculate, view, and analyze the energy levels of adiabatic Stark energy curves of linear, symmetric top and asymmetric top molecules. The program exploits the symmetry of the Hamiltonian to generate fully labeled adiabatic Stark energy curves.

CMISTARK is written in Python and easily extendable, while the core numerical calculations make use of machine optimized BLAS and LAPACK routines. Calculated energies are stored in HDF5 files for convenient access and programs to extract ASCII data or to generate graphical plots are provided.
\end{abstract}

\section{Program summary}

Program title: CMIstark

Catalogue identifier: AEQS_v1_0

Program summary URL: http://cpc.cs.qub.ac.uk/summaries/AEQS_v1_0.html

Program obtainable from: CPC Program Library, Queen's University, Belfast, N. Ireland

Licensing provisions: GNU General Public License version 3 or later with amendments. See code for details.

No. of lines in distributed program, including test data, etc.: 4091

No. of bytes in distributed program, including test data, etc.: 62805

Distribution format: tar.gz

Programming language: Python (version 2.6.x, 2.7.x).

Computer: Any Macintosh, PC, or Linux/UNIX workstations with a modern Python distribution.

Operating system: Tested on Mac OS X and a variety of Linux distributions.

RAM: 2 GB for typical calculations

Classification: 16.1.

External routines:

Python packages numpy and scipy; utilizes (optimized) LAPACK and BLAS through scipy. All packages available under open-source licenses.

\footnotetext{
th This paper and its associated computer program are available via the Computer Physics Communication homepage on ScienceDirect (http://www.sciencedirect.com/ science/journal/00104655).

* Corresponding author at: Center for Free-Electron Laser Science, DESY, Notkestrasse 85, 22607 Hamburg, Germany. E-mail address: jochen.kuepper@cfel.de (J. Küpper). URL: http://desy.cfel.de/cid/cmi (J. Küpper).

1 Present address: Bruker AXS GmbH, Karlsruhe, Germany.
} 
Nature of problem:

Calculation of the Stark effect of asymmetric top molecules in arbitrarily strong dc electric fields in a correct symmetry classification and using correct labeling of the adiabatic Stark curves.

Solution method:

We set up the full $M$ matrices of the quantum-mechanical Hamiltonian in the basis set of symmetric top wavefunctions and, subsequently, Wang transform the Hamiltonian matrix. We separate, as far as possible, the sub-matrices according to the remaining symmetry, and then diagonalize the individual blocks. This application of the symmetry consideration to the Hamiltonian allows an adiabatic correlation of the asymmetric top eigenstates in the dc electric field to the field-free eigenstates. This directly yields correct adiabatic state labels and, correspondingly, adiabatic Stark energy curves.

Restrictions:

The maximum value of $J$ is limited by the available main memory. A modern desktop computer with 16 GB of main memory allows for calculations including all $J s$ up to a values larger than 100 even for the most complex cases of asymmetric tops.

Running time:

Typically $1 \mathrm{~s}-1$ week on a single CPU or equivalent on multi-CPU systems (depending greatly on system size and RAM); parallelization through BLAS/LAPACK. For instance, calculating all energies up to $J=25$ of indole (vide infra) for one field strength takes 1 CPU-s on a current iMac.

(c) 2013 Elsevier B.V. All rights reserved.

\section{Introduction}

Over the last decade, the manipulation of the motion of molecules using electric fields has been revitalized [1-5]. Exploiting the Stark effect, large asymmetric-top polar molecules have been deflected [6], focused [7], and decelerated [8]. These techniques can be used to spatially separate neutral molecules according to their quantum states [9], structural isomers [7,10], and cluster sizes [11]. These techniques promise advanced applications of well-defined samples of complex molecules in various research fields, e.g., modern spectroscopies [12,13] or the direct imaging of structural and chemical dynamics [2,14-16]. However, successful implementation of these methods requires a thorough theoretical understanding of the molecule-field interaction for the involved molecular quantum states. Here we provide a well-tested and optimized program package for the calculation and labeling of so called Stark curves, i.e., the energies of molecules as a function of electric field strength, for general use. This software package will benefit the advance of those forthcoming applications, esp. also for complex molecules. Moreover, it allows non-specialists and newcomers to the field to concentrate on their envisioned applications of controlled molecules.

The code presented here is designed to calculate eigenenergies of very cold (on the order of a few Kelvin) ensembles of polar molecules in the presence of external electrostatic fields. The interaction of the molecular dipole moment with the dc electric field changes the internal energy, and this is called the Stark effect. To quantify this behavior, the eigenvalue problem of the Hamiltonian is solved. CMIsTARK does this calculation in terms of numerically diagonalizing the corresponding Hamiltonian matrix. An efficient method of diagonalizing the matrix, exploiting underlying physics phenomena, is employed. Moreover, a correct method of correlating eigenvalues to quantum states, i. e., labeling the calculated energies for all field strengths, is also required for further use in order to predict or simulate and analyze control experiments.

The software package is named CMIsTARK. It is developed and maintained by the Controlled Molecule Imaging (CMI) group at the Center for Free Electron Laser Science (CFEL), DESY, in Hamburg, based on earlier work by some of the authors at the Fritz Haber Institute of the MPG in Berlin.

\section{Description}

Stark energies are obtained by setting up and diagonalizing the Hamiltonian matrix for a given electric field strength. The matrix elements can be obtained analytically (vide infra) and the resulting matrix is diagonalized numerically to obtain its eigenvalues, corresponding to the energies of the molecular states. First, the matrix is block-diagonalized as far as possible using symmetry considerations in order to correctly assign quantum numbers to eigenvalues. The block-diagonalization also significantly reduces the overall computation time, which is dominated by the diagonalization. The resulting blocks are diagonalized using LAPACK's dsyevr or zheevr subroutines for real and complex matrices, respectively. The following overview section will provide a brief review of the main concepts of the above approach.

\subsection{Overview}

The quantum-mechanical energy of a molecule, $E$, can be obtained by solving the Schrödinger equation

$$
H \Psi=E \Psi .
$$

Neglecting translation, $H$ denotes the Hamiltonian operator in the center-of-mass frame and $\Psi$ is the wavefunction. For a rigid rotor and neglecting nuclear hyperfine-structure effects, the Hamiltonian can be expressed in terms of components of the total angular moment operator $\mathbf{J}$ about the principal axes $(a, b, c)$, i. e., $\mathbf{J}_{a}, \mathbf{J}_{b}, \mathbf{J}_{c}[17,18]$ :

$$
H_{\text {rigid }}=\hbar^{2}\left(\frac{\mathbf{J}_{a}^{2}}{2 I_{a}}+\frac{\mathbf{J}_{b}^{2}}{2 I_{b}}+\frac{\mathbf{J}_{c}^{2}}{2 I_{c}}\right)=h\left(A \mathbf{J}_{a}^{2}+B \mathbf{J}_{b}^{2}+C \mathbf{J}_{c}^{2}\right),
$$


Table 1

Types of rotors defined through their inertial parameters.

\begin{tabular}{lll}
\hline Moments of inertia & Rotational constant & Rotor type \\
\hline$I_{a}=0 ; I_{b}=I_{c}$ & $A=\infty, B=C$ & Linear top \\
$I_{a}=I_{b}=I_{c}$ & $A=B=C$ & Spherical top \\
$I_{a}<I_{b}=I_{c}$ & $A>B=C$ & Prolate symmetric top \\
$I_{a}=I_{b}<I_{c}$ & $A=B>C$ & Oblate symmetric top \\
$I_{a} \neq I_{b} \neq I_{c}$ & $A \neq B \neq C$ & Asymmetric top \\
\hline
\end{tabular}

where $h$ is Planck's constant, $\hbar=h / 2 \pi$, and $I_{a}, I_{b}$ and $I_{c}$ are three principal moments of inertia of the rotor. By convention, the principal axes of inertia $(a, b, c)$ are labeled such that $I_{a} \leq I_{b} \leq I_{c}$. Note that, in the program, instead of moments of inertia we use rotational constants, $A, B, C$, which in units of $\operatorname{Hertz}(\mathrm{Hz})$ are $[17,18]$ :

$$
A=\frac{h}{8 \pi^{2} I_{a}}, \quad B=\frac{h}{8 \pi^{2} I_{b}}, \quad C=\frac{h}{8 \pi^{2} I_{c}} .
$$

Molecular rotors are classified in terms of the magnitudes of their inertial moments, or rotational constants, as shown in Table 1. Several quantum numbers are used to denote zero-field wavefunctions and energies of the rotational states of molecules [17,18]. The Schrödinger equation of $H_{\text {rigid }}$, (1) and (2), of linear rotors and symmetric tops in free space (see Table 1) can be solved analytically, and their eigenfunctions of $H_{\text {rigid }}$ are expressed as spherical harmonics $|J, M\rangle$ and Wigner $D$ matrices $|J, K, M\rangle$, respectively [17,18]. $J$ represents the quantum number of total angular momentum $\mathbf{J}, K$ characterizes the projection of $\mathbf{J}$ onto the symmetry axis of the symmetric top, and $M$ is the quantum number characterizing the projection of $\mathbf{J}$ onto a space fixed $Z$-axis. For asymmetric tops $K$ is not a good quantum number and the Schrödinger equation of $H_{\text {rigid }}$ cannot generally be solved analytically. A numerical calculation uses symmetric top wavefunctions $|J, K, M\rangle$ as a basis set for obtaining asymmetric top eigenfunctions $\left|J_{K_{a} K_{c}} M\right\rangle$. Here, the quantum number $J$ and two pseudo quantum numbers $K_{a}, K_{c}$ specify the zero-field rotational states. Finally, we only focus on closed shell molecules, i. e., molecules which do not have unpaired electrons. Therefore, the values of $J, K$ and $M$ are integers.

However, a real molecular system is not rigid. It is assumed that all non-rigidity under the experimental conditions (on the order of $1 \mathrm{~K}$ ) can be described by a Hamiltonian representing centrifugal distortion, $H_{\mathrm{d}}$, with corresponding centrifugal distortion constants [17]. The Hamiltonian for such a non-rigid rotor is thus written as: $H_{\text {rot }}=H_{\text {rigid }}+H_{\mathrm{d}}$. Further details of $H_{\mathrm{d}}$ for each type of rotor are described in the following sections. In all cases we have implemented the lowest order quartic centrifugal distortion terms. Higher order terms can easily be added if necessary.

The Stark effect of a polar molecule in a dc electric field is dominated by the interaction $\vec{\mu} \cdot \vec{\varepsilon}$ of the molecule's dipole moment $\vec{\mu}$ with the field $\vec{\varepsilon}$. While higher-order effects become relevant in strong fields, they can still be neglected in our case. For instance, the permanent dipole moment of benzonitriles ground state leads to an energy shift of $300 \mathrm{GHz}$ at $200 \mathrm{kV} / \mathrm{cm}$, but the corresponding effect due to the polarizability of the very similar non-polar molecule benzene is only $50 \mathrm{MHz}$ [19], i. e., almost four orders of magnitude smaller.

The dipole interaction with the electric field is described by the following contribution to the Hamiltonian:

$$
H_{\text {Stark }}=-\varepsilon \sum_{g=x, y, z} \mu_{g} \phi_{Z g}
$$

where $x, y, z$ represent a molecule-fixed coordinate system, $\mu_{g}$ represent the dipole moment components along the molecule-fixed axes $x, y, z$, and $\phi_{Z_{g}}$ are the direction cosines of the $x, y, z$ axes with reference to the space-fixed $X, Y, Z$-axes. $Z$ is oriented along the electricfield direction. In the program, the principle axis system $(a, b, c)$ is identified with the molecule-fixed system $(x, y, z)$ in representation $I^{r}(x=b, y=c, z=a)[17,18]$. Note that this definition has the advantage that the Stark Hamiltonian does not mix states with different values of $K$ if the dipole moment is parallel to the molecular $a$ axis. The rotational Hamiltonian in the field $\varepsilon$ can thus be written as: $H_{\text {rot, } \varepsilon}=H_{\text {rot }}+H_{\text {Stark }}$.

In the program, the Schrödinger equation of the Hamiltonian in the field, $H_{\text {rot, } \varepsilon}$, is solved numerically. The corresponding Hamiltonian matrix and the strategy of its diagonalization are described in following sections for each type of rotor. Finally, the program assigns the calculated rotational energies in the field to "adiabatic quantum numbers", i. e., to the adiabatically corresponding field-free rotor states [20]. To ensure correct assignments, a symmetry classification of $H_{\mathrm{rot}, \varepsilon}$ and quantum states according the electric field symmetry group [21,22] is required. In addition to the separation of $M$ and, for the symmetric top, $K$, this is achieved through an appropriate unitary transformation of $H_{\text {rot, } \varepsilon}$ following Wang's method [17,23].

\subsection{Linear top}

In a linear polyatomic molecule, the moment of inertia about the principal axis $a$ is zero whereas the two other moments of inertia along axes $b$ and $c$ are equal: $I_{b}=I_{c}=I$. The centrifugal distortion Hamiltonian $H_{\mathrm{d}}$ takes the form

$$
H_{\mathrm{d}}=-h D \mathbf{J}^{4}
$$

where $D(\mathrm{~Hz})$ is a centrifugal distortion constant. The first-order perturbation energy, which is the expectation value of $H_{\mathrm{d}}$ over field-free linear rotor wavefunctions, $|J, M\rangle$, is included in the Hamiltonian matrix. The dipole moment of a linear molecule is along its symmetry axis $z$, i. e., $\mu_{z}=\mu$ and $\mu_{x}=\mu_{y}=0$. Thus the Stark Hamiltonian simply becomes

$$
H_{\text {Stark }}=-\mu \varepsilon \phi_{Z_{z}}
$$

The non-zero matrix elements for $H_{\text {rot }}$ and $H_{\text {Stark }}$ in the basis of linear top wavefunctions $|J, M\rangle$, are provided in Appendix A. The Hamiltonian matrix is diagonalized directly without any further simplification. 


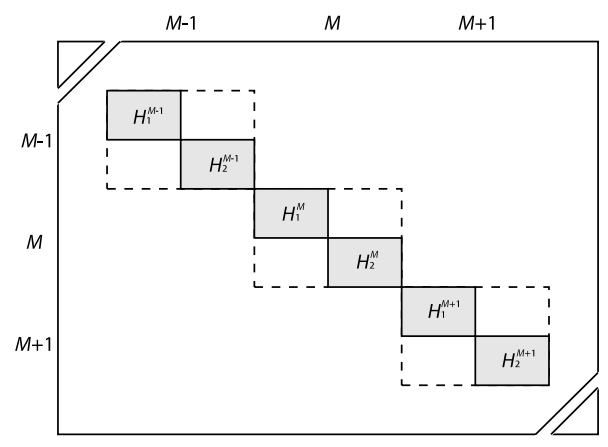

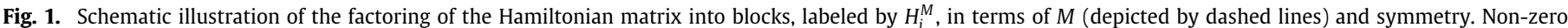

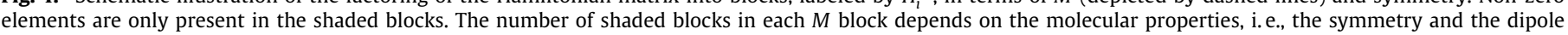
moment direction; see text for details.

\subsection{Symmetric top}

A molecule in which two of the principal moments of inertia are equal is a symmetric top rotor, such as a prolate top $\left(I_{a}<I_{b}=I_{c}\right)$ and an oblate top $\left(I_{a}=I_{b}<I_{c}\right)$. The figure axis of the molecule, ${ }^{2}$ which is parallel to the dipole moment, must lie along the special principal axis of inertia, i. e., along the $a$ axis for a prolate top and the $c$ axis for an oblate top. The non-rigidity of a symmetric top is taken into account by including the first order perturbation energy of the corresponding centrifugal distortion Hamiltonian $\left(H_{\mathrm{d}}\right)$ [17] into the Hamiltonian matrix. The Stark Hamiltonian $H_{\text {Stark }}$ of symmetric tops is the same as that for linear rotors, as shown in Eq. (6). The matrix elements for $H_{\text {rot }}$ and $H_{\text {Stark }}$ in the basis of symmetric top wavefunctions, $|J, K, M\rangle$, are listed in Appendix B. Finally, the strategy for diagonalizing symmetric and asymmetric top Hamiltonian matrices is the same, and is described in the next section. Moreover, in the case of the symmetric top, $K$ is a good quantum number and an additional factorization into separate $K$ blocks is possible.

\subsection{Asymmetric top}

An asymmetric top molecule has three non-zero and non-equal principal moments of inertia. As mentioned before, its Schrödinger equation even in the field-free case has no trivial analytical solution for general $J$, and field-free symmetric top wavefunctions, $|J, K, M\rangle$, are used as the basis set for the Hamiltonian matrix $H_{\mathrm{rot}, \varepsilon}$ [17]. All non-zero matrix elements of $H_{\mathrm{rot}, \varepsilon}$ in this basis set are listed in Appendix C. Note that, in this Hamiltonian matrix $H_{\mathrm{rot}, \varepsilon}$, there are no off-diagonal matrix elements in $M$, because $M$ is still a good quantum number in the field. Thus, the blocks of each value of $M$ in the matrix, as shown in Fig. 1, can be diagonalized separately. As $K$ in the asymmetric top case and $J$ in the non-zero field case are not good quantum numbers the set of basis functions for the block must cover a wide enough range of $K$ and $J$ to ensure the accuracy of the numerical solution of the eigenvalue problem.

A further simplification of the matrix can be obtained by considering the symmetry properties of the Hamiltonian. As mentioned before, the symmetry classification is required in order to distinguish avoided crossings (between curves with the same symmetry) and real crossings (between curves with different symmetries) and to assign the energy levels correctly. The field-free Hamiltonian operator $\left(H_{\text {rigid }}\right.$ and $H_{\text {rot }}$ ) belongs to a symmetry group called Fourgroup and it is designated by $V(a, b, c)$ (see Appendix D for a detailed introduction). However, symmetric top wavefunctions, which are the natural basis set, do not belong to the Fourgroup. A transformation to a symmetrized basis is provided by the Wang transformation of the Hamiltonian matrix [17]:

$$
H_{\text {Wang }}=\widetilde{X} H X=\sum_{i} H_{i}
$$

where $X$ denotes the Wang transformation matrix and $H_{i}$ denotes a sub-matrix for each symmetry species $i$. Thus, the Hamiltonian is expressed in a basis of linear combinations of symmetric top wavefunctions which obey the Fourgroup symmetry [17]. For a fieldfree asymmetric top, its Wang transformed Hamiltonian matrix, i. e., $H_{\text {Wang, rot }}$, can be factorized into four sub-matrices in terms of the Fourgroup symmetry species, as described in Appendix D.

When an external field is applied, the number of sub-matrices in the Hamiltonian matrix, $H_{\text {Wang, rot, } \varepsilon}$, usually reduces depending on the dipole moment direction in the molecule and on the values of $M$. As described in Appendices $E$ and $F$, if the molecule's dipole moment is parallel to one principal axis of inertia, $H_{\text {Wang, rot, } \varepsilon}$ can be factorized into two sub-matrices for $M \neq 0$, and four for the special case $M=0$. If the molecule's dipole moment is not parallel to any principal axis of inertia, no factorization of $H_{\mathrm{Wang}}$, rot, $\varepsilon$ is possible for $M \neq 0$. For $M=0$ a factorization into two blocks is still possible if one dipole moment component $\mu_{\alpha}(\alpha=a, b, c)$ is zero. In any case, the above block diagonalization ensures that all eigenstates obtained from the diagonalization of each sub-matrix $H_{i}^{M}$ (see Fig. 1 ) belong to the same symmetry. This means that all crossings between eigenstates of each sub-matrix are avoided and that the energy order of these states remains the same adiabatically, at any field strength. As a result, we can sort the resulting states of each $H_{i}^{M}$ by energy and assign quantum number labels in the same order as for energy-sorted field-free states of the same symmetry. This yields a correct assignment of "adiabatic quantum number labels", $\tilde{J}_{\tilde{K}_{a} \tilde{K}_{c}} \tilde{M}$, to rotational states in the field [20].

\footnotetext{
2 Here we consider axially symmetric molecules, ignoring molecules which accidentally have an equivalent tensor of inertia.
} 

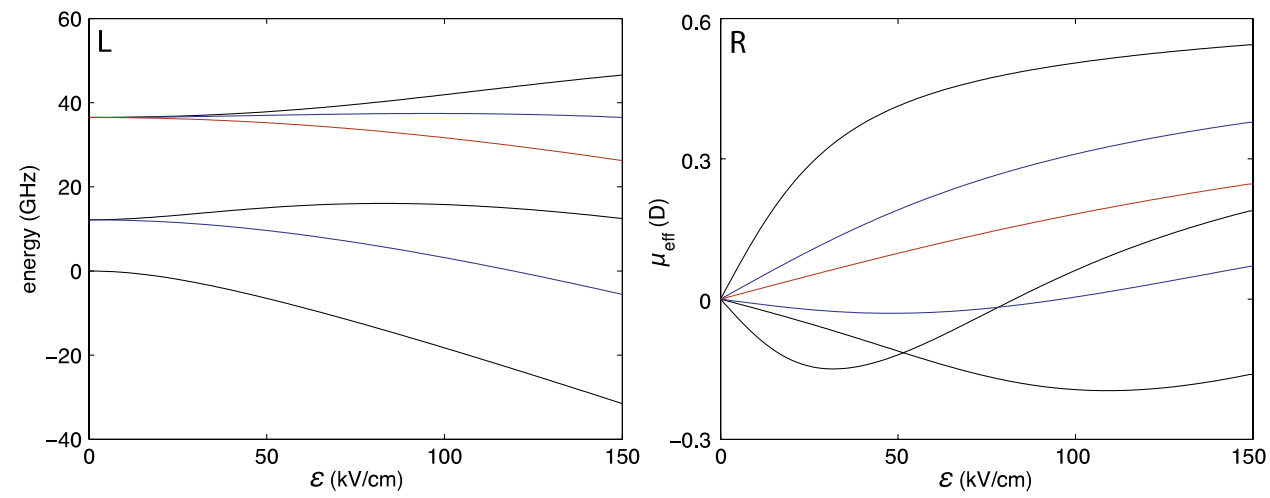

(a) OCS.
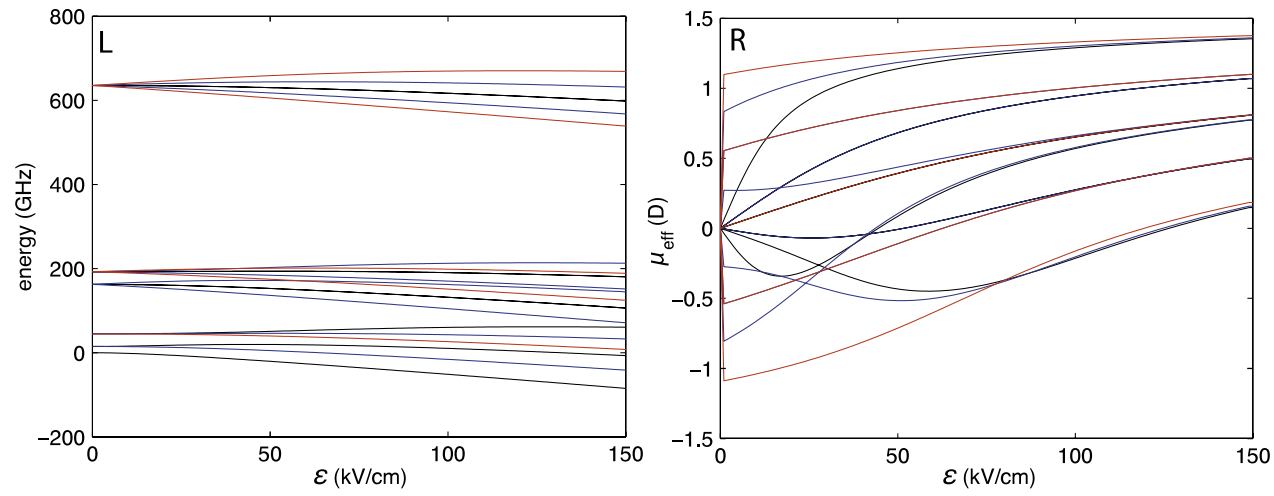

(b) Iodomethane.
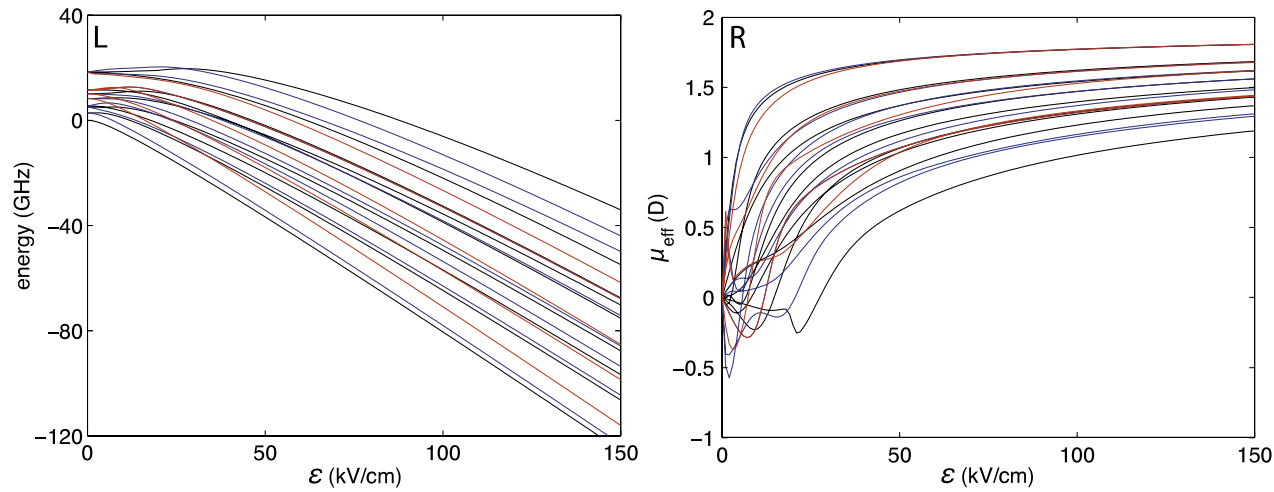

(c) Indole.

Fig. 2. (L) Stark energies and (R) effective dipole moments ( $\mu_{\text {eff }}$ ) of OCS, iodomethane, and indole for the $M=0$ (black), $M=1$ (blue), and $M=2$ (red) levels of $J=0-2$. (For interpretation of the references to colour in this figure legend, the reader is referred to the web version of this article.)

\subsection{Results}

In practice, the calculation of the Stark energies is performed for a number of electric field strengths - typically in steps of $1 \mathrm{kV} / \mathrm{cm}$ from $0 \mathrm{kV} / \mathrm{cm}$ to $200 \mathrm{kV} / \mathrm{cm}$ - and the resulting energies are stored for later use. The calculated Stark curves and effective dipole moments for lowest-lying rotational quantum states of OCS (linear rotor), iodomethane (symmetric top), and indole (asymmetric top) are plotted using cmistark_plot_energies and are shown in Fig. 2(a), (b), (c), respectively. Here the effective dipole moment, $\mu_{\text {eff, is }}$ introduced as:

$$
\mu_{\mathrm{eff}}(\varepsilon)=-\frac{\partial E(\varepsilon)}{\partial \varepsilon}
$$

This is the space fixed dipole moment, i.e., the projection of the molecular frame dipole moment onto the field direction. It is extremely useful in further simulations on the manipulation of polar molecules with inhomogeneous electric fields, where the force exerted on the molecule is directly proportional to $\mu_{\text {eff }}[24]$.

Firstly, in these figures, the Stark energy curves from different $M$ always cross, i. e., $M$ is a good quantum number. Secondly, while the energy curves of OCS (Fig. 2(a) L) and iodomethane (Fig. 2(b) L) show relatively simple structures, those for indole (Fig. 2(c) L) show a more complicated behavior. Furthermore, for curves of indole of each $M$ (Fig. 2(c) L), most crossings between Stark curves are avoided, because 
the dipole moment of indole is not parallel to any principal axis ( $\left.\mu_{a} \neq 0, \mu_{b} \neq 0, \mu_{c}=0\right)$. Thus, for $M \neq 0$ all states in the field have the same symmetry. For OCS and iodomethane, the sign of the effective dipole moment, $\mu_{\text {eff, }}$ can be negative or positive, depending on the quantum state and field strength, as shown in Fig. 2(a) R and (b) R. However, for indole, the sign of $\mu_{\text {eff }}$ is mostly positive, as shown in Fig. 2(c) R. The rapid changes of signs and values of $\mu_{\mathrm{eff}}$ shown in Fig. 2(c) R are due to avoided crossings between Stark curves.

In order to evaluate the performance, we have calculated the energy curves for OCS at 151 field strengths in the range 0 to $150 \mathrm{kV} / \mathrm{cm}$ using different algorithms for three types of rotors for all states up to $J=32$. This yields the following computation times on a current iMac:

- Linear rotor code: $1.0 \mathrm{~s}$

- Symmetric top code: $55 \mathrm{~s}$

- Asymmetric top code: 300 s (3 min 20 s, 150\% CPU utilization).

Note that the runtime largely reflects the time spent on diagonalizing the matrix, and thus the size of the matrix. According to the LAPACK benchmark report in LAPACK Users' Guide [25], for diagonalizing dense symmetric $N$ by $N$ matrices by using dsyevr, the computing time for $N=1000$ is about 400 times of that for $N=100$. However, the computing time for $N=2000$ is about 10 times of that for $N=1000$. In practice, for asymmetric top calculations an increase of the maximum $J$ by 10 (e.g., $J=40 \rightarrow 50$ or $J=90 \rightarrow 100$ ) included in the calculation roughly doubles the runtime.

\section{Installation instructions}

\subsection{Requirements}

CMIsTARK needs an operational Python installation, the external Python packages, numpy, scipy, PyTables, matplotlib, and a commandline interface to start the various python scripts provided here.

\subsection{Obtaining the code}

The program is available from the CPC Program Library, Queen's University, Belfast, N. Ireland. The latest version of the program can also be obtained from the Controlled Molecule Imaging (CMI) group.

\subsection{Installation}

Installation is performed by executing the generic Python install command python setup.py install in the unpacked source code directory. This requires write access to the packages directory tree of the Python distribution. Alternatively, on Unix-like systems the provided shell-script user-install . sh can be used to install the program into an user-specified directory, such as \$HOME/ . python. This method requires the user to define the shell variable PYTHONHOME to include this directory in the python search path.

\section{Documentation}

A full documentation is provided within the source code and only briefly summarized here. To perform a Stark effect calculation the script file cmistark_calculate_energy is used. Some of its general command-line options are

- --<moleculename $>$ : specify which molecule is used in the calculation,

- --dc-fields: specify the range of the dc electric field strength,

- --Jmax_calc: specify the maximum value of $J$ included in the calculation,

- --Jmax_save: specify the maximum value of $J$ of Stark curves saved in the output file.

Two scripts cmistark_plot_energy and cmistark_print_energy are provided to access existing files with stored Stark curves, and plot or convert to text, respectively, all or selected Stark energy curves.

Calculating the Stark curves of OCS is as simple as running the command

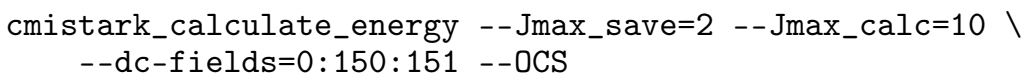

The data is saved in OCS.molecule and no command-line output is produced. The correct output resulting from this calculation is provided in the samples / directory of the source code. The plot in Fig. 2(a) L can then be produced by running

$$
\text { cmistark_plot_energy OCS.molecule }
$$

Currently, isotopologues of the following molecules are implemented in cmistark_calculate_energy, with parameters from the literature as referenced in the code: 3-aminophenol, carbonylsulfide, water, indole, indole(water) ${ }_{1}$, iodomethane, difluoroiodobenzene, aminobenzonitrile, benzonitrile, iodobenzene, and sulfurdioxide. Implementing a new molecule is as simple as adding a code block in cmistark_calculate_energy to define relevant molecular parameters, molecular constants and dipole moment components. For the cis and trans conformers of 3-aminophenol [26], this is implemented in the following way: 
def three_aminophenol(param):

\# Inertial parameters: Filsinger et al, Phys. Chem. Chem. Phys. 10, 666 (2008)

\# dipole moment: Filsinger et al, Phys. Chem. Chem. Phys. 10, 666 (2008) param.name = "3-aminophenol"

param.watson = 'A' \# specify Watson's A reduction

param.symmetry = 'N' \# specify "no symmetry" in the dc electric field

if param.isomer $==0$ : \# cis-conformer

\# the following line specifies the rotational constants (A, B, C)

\# in unit of $\mathrm{Hz}$.

param.rotcon $=$ convert. Hz2J (numpy. $\operatorname{array}([3734.93 e 6,1823.2095 e 6,1226.493 e 6]))$

\# the following line specifies the dipole moment components $\left(\$ \backslash m u_{-} a, \backslash m u_{-} b, \backslash m u_{-} c \$\right)$

\# in unit of Debye.

param.dipole $=$ convert. $\mathrm{D} 2 \mathrm{Cm}($ numpy. $\operatorname{array}([1.7718,1.517,0])$.

elif param.isomer $==1$ : \# trans-conformer

param.rotcon $=$ convert.Hz2J (numpy. $\operatorname{array}([3730.1676 \mathrm{e} 6,1828.25774 \mathrm{e} 6,1228.1948 \mathrm{e} 6]))$

param.dipole $=$ convert.D2Cm (numpy.array $([0.5563,0.5375,0])$.

\section{Alternative software}

Several programs exist for the simulation of rotationally resolved spectra of asymmetric top molecules, such as PGOPHER [27], SPFIT/SPCAT [28,29], QSTARK [30-32], DBSROT [33,34], KROT [35], ASYROT [36] and JB95 [37], as well as programs for automated fitting of high resolution spectra, e.g., based on genetic algorithm [38]. Inherently these programs work by calculating the energies of all states possibly involved in the relevant transitions, i. e., they do perform similar calculations as CMIsTARK. So far, to the best of our knowledge, only the programs PGOPHER, DBSROT, and QSTARK can also calculate Stark energies of linear, symmetric, and asymmetric rotors. The program QSTARK [30-32] allows calculations including quadrupole coupling effects for one nucleus. PGOPHER [27] can take into account some internal motions, such as internal rotations (torsion) or inversion motions, e.g., in $\mathrm{NH}_{3}$. These effects will be implemented in future versions of CMISTARK. However, they require considerably more intricate handling of symmetry properties. The available programs are not well suited for simulations in the controlled molecules field where it is necessary to calculate Stark energies in very strong fields and to correctly label large numbers of quantum states over the full field-strength regime. For example, while the program QSTARK calculates Stark energies essentially correctly in strong fields, labeling problems are known when the off-diagonal elements in the $H$ matrix become sufficiently large [32]. PGOPHER provides direct access to the Stark curves of individual or a few quantum states. However, its graphical/text based access is not convenient for the calculation and storage of many precisely calculated Stark curves with a sufficiently large range of quantum states. Note that, even for relatively small complex molecules, such as benzonitrile or indole under conditions of only a few K, many thousand Stark curves need to be calculated with $J$ up to 50, with hundreds of energies per curve for specific dc field strengths, and they must be stored for easy retrieval in further calculations.

\section{Outlook}

The current program has been successfully used in the calculation of Stark energy maps of various asymmetric top molecules, for instance, benzonitrile [8], 4-aminobenzonitrile [39], 3-aminophenol [7,10], indole, and indole-water clusters [11]. Those calculation results from the program were successfully applied to fit and analyze experimental data on the manipulation of molecules with electric fields. The program was also tested against the energies of lowest rotational states from QSTARK [30-32], with a relative error of the order of $10^{-6}$ limited by the numerical precision of slightly different implementations of the Hamiltonian and the matrix diagonalization.

The current program will be further improved in several directions. For example, for molecules containing large nuclear quadrupole constants the corresponding quadrupole coupling terms need to be implemented. The challenge here is to still automatically symmetrize the Hamiltonian and to correctly label the resulting states. Moreover, especially many of the small molecules employed in electric-field manipulation experiments are open-shell, i. e., they possess electronic (orbital and spin) angular momentum. The respective Hamiltonians could also be implemented in CMISTARK. We will implement such extensions as they are relevant for the simulation of our manipulation experiments. We will support third parties to extend our code to their needs, under the provision that it is provided to all users after a reasonable amount of time.

\section{Acknowledgments}

We thank Rosario González-Férez, Bas van der Meerakker, Gerard Meijer, and members of the CFEL-CMI group for helpful discussions. Izan Castro Molina implemented the initial linear and symmetric top calculations. This work has been supported by the DFG priority program 1116 "Interactions in ultracold atomic and molecular gases" and by the excellence cluster "The Hamburg Center for Ultrafast Imaging - Structure, Dynamics and Control of Matter at the Atomic Scale” of the Deutsche Forschungsgemeinschaft.

\section{Appendix A. Matrix elements for linear rotors}

For the linear top, the matrix elements of $H_{\text {rigid }}$ and $H_{\mathrm{d}}$ can be written as [17]:

$$
\begin{aligned}
& \left\langle J, M\left|H_{\text {rigid }}\right| J, M\right\rangle=h B J(J+1), \\
& \left\langle J, M\left|H_{\mathrm{d}}\right| J, M\right\rangle=-h D J^{2}(J+1)^{2},
\end{aligned}
$$


where $B(\mathrm{~Hz})$ and $D(\mathrm{~Hz})$ are the corresponding rotational constant and the quartic centrifugal distortion constant, respectively. The matrix elements for the Stark Hamiltonian $H_{\text {Stark }}$ are expressed as follows [18]:

$$
\left\langle J+1, M\left|H_{\text {Stark }}\right| J, M\right\rangle=\left\langle J, M\left|H_{\text {Stark }}\right| J+1, M\right\rangle=-\frac{\sqrt{(J+1)^{2}-M^{2}}}{\sqrt{(2 J+1)(2 J+3)}} \mu \varepsilon .
$$

\section{Appendix B. Matrix elements for symmetric tops}

For the rigid prolate and oblate symmetric top the matrix elements of $H_{\text {rigid }}$ can be written as [17]:

$$
\begin{array}{ll}
\left\langle J, K, M\left|H_{\text {rigid }}\right| J, K, M\right\rangle=h\left[B J(J+1)+(A-B) K^{2}\right] & \text { (prolate) } \\
\left\langle J, K, M\left|H_{\text {rigid }}\right| J, K, M\right\rangle=h\left[B J(J+1)+(C-B) K^{2}\right] & \text { (oblate) }
\end{array}
$$

with the rotational constants $A, B, C(\mathrm{~Hz})$. The matrix elements of $H_{\mathrm{d}}$ are expressed as following [17]:

$$
\left\langle J, K, M\left|H_{\mathrm{d}}\right| J, K, M\right\rangle=-h\left[\Delta_{J} J^{2}(J+1)^{2}+\Delta_{J K} J(J+1) K^{2}+\Delta_{K} K^{4}\right]
$$

where $\Delta_{J}, \Delta_{J K}$ and $\Delta_{K}$ are the first-order (quartic) centrifugal distortion constants (Hz). The matrix elements of $H_{\text {Stark }}$ are [18]:

$$
\begin{aligned}
& \left\langle J, K, M\left|H_{\text {Stark }}\right| J, K, M\right\rangle=-\frac{M K}{J(J+1)} \mu \varepsilon \\
& \left\langle J+1, K, M\left|H_{\text {Stark }}\right| J, K, M\right\rangle=\left\langle J, K, M\left|H_{\text {Stark }}\right| J+1, K, M\right\rangle=-\frac{\sqrt{(J+1)^{2}-K^{2}} \sqrt{(J+1)^{2}-M^{2}}}{(J+1) \sqrt{(2 J+1)(2 J+3)}} \mu \varepsilon .
\end{aligned}
$$

\section{Appendix C. Matrix elements for asymmetric tops}

For the rigid asymmetric top, the matrix elements of $H_{\text {rigid }}$ in terms of $I^{r}$ representation [17] can be written as [17,18]:

$$
\begin{aligned}
& \left\langle J, K, M\left|H_{\text {rigid }}\right| J, K, M\right\rangle=h\left[\frac{B+C}{2}\left(J(J+1)-K^{2}\right)+A K^{2}\right] \\
& \left\langle J, K+2, M\left|H_{\text {rigid }}\right| J, K, M\right\rangle=\left\langle J, K, M \mid H_{\text {rigid }} J, K+2, M\right\rangle=\frac{h(B-C)}{4} \sqrt{J(J+1)-K(K+1)} \sqrt{J(J+1)-(K+1)(K+2)},
\end{aligned}
$$

with the rotational constants $A, B, C(\mathrm{~Hz})$. The distortable rotor is described using Watson's A reduction [40]:

$$
\begin{aligned}
\left\langle J, K, M\left|H_{\mathrm{d}}\right| J, K, M\right\rangle=-h & {\left[\Delta_{J}(J(J+1))^{2}+\Delta_{J K} J(J+1) K^{2}+\Delta_{K} K^{4}\right], } \\
\left\langle J, K+2, M\left|H_{\mathrm{d}}\right| J, K, M\right\rangle= & \left\langle J, K, M \mid H_{\mathrm{d}} J, K+2, M\right\rangle \\
= & -h\left[\delta_{J} J(J+1)+\frac{\delta_{K}}{2}\left((K+2)^{2}+K^{2}\right)\right] \sqrt{J(J+1)-K(K+1)} \sqrt{J(J+1)-(K+1)(K+2)}
\end{aligned}
$$

with the five linearly independent quartic distortion constants $\Delta_{J}, \Delta_{J K}, \Delta_{K}, \delta_{J}$ and $\delta_{K}(\mathrm{~Hz})$. The contribution of $\mu_{a}$, i.e., the dipole moment component along the principal axis of inertia $a$, is $[18,41]$ :

$$
\begin{aligned}
& \left\langle J, K, M\left|H_{\text {Stark }}^{a}\right| J, K, M\right\rangle=-\frac{M K}{J(J+1)} \mu_{a} \varepsilon \\
& \left\langle J+1, K, M\left|H_{\text {Stark }}^{a}\right| J, K, M\right\rangle=\left\langle J, K, M\left|H_{\text {Stark }}^{a}\right| J+1, K, M\right\rangle=-\frac{\sqrt{(J+1)^{2}-K^{2}} \sqrt{(J+1)^{2}-M^{2}}}{(J+1) \sqrt{(2 J+1)(2 J+3)}} \mu_{a} \varepsilon .
\end{aligned}
$$

The contribution of $\mu_{b}$ is:

$$
\begin{aligned}
& \left\langle J, K+1, M\left|H_{\text {Stark }}^{b}\right| J, K, M\right\rangle=-\frac{M \sqrt{(J-K)(J+K+1)}}{2 J(J+1)} \mu_{b} \varepsilon \\
& \left\langle J+1, K \pm 1, M\left|H_{\text {Stark }}^{b}\right| J, K, M\right\rangle= \pm \frac{\sqrt{(J \pm K+1)(J \pm K+2)} \sqrt{(J+1)^{2}-M^{2}}}{2(J+1) \sqrt{(2 J+1)(2 J+3)}} \mu_{b} \varepsilon .
\end{aligned}
$$

The $H_{\text {Stark }}$ matrix elements involving $\mu_{c}$ are:

$$
\begin{aligned}
& \left\langle J, K \pm 1, M\left|H_{\text {Stark }}^{c}\right| J, K, M\right\rangle= \pm i \frac{M \sqrt{(J \mp K)(J \pm K+1)}}{2 J(J+1)} \mu_{c} \varepsilon \\
& \left\langle J+1, K \pm 1, M \mid H_{\text {Stark }}^{c} J, K, M\right\rangle=-i \frac{\sqrt{(J \pm K+1)(J \pm K+2)} \sqrt{(J+1)^{2}-M^{2}}}{2(J+1) \sqrt{(2 J+1)(2 J+3)}} \mu_{c} \varepsilon .
\end{aligned}
$$

Note that the equations above use the representation $I^{r}$ with the phase convention and formalism of Zare [18]. 
Table D.2

Character table for the four group V.

\begin{tabular}{llrrr}
\hline $\mathrm{V}$ & $\mathrm{E}$ & $C_{2}^{a}$ & \multicolumn{1}{c}{$C_{2}^{b}$} & \multicolumn{1}{c}{$C_{2}^{c}$} \\
\hline $\mathrm{A}$ & 1 & 1 & 1 & 1 \\
$\mathrm{~B}_{a}$ & 1 & 1 & -1 & -1 \\
$\mathrm{~B}_{b}$ & 1 & -1 & 1 & -1 \\
$\mathrm{~B}_{c}$ & 1 & -1 & -1 & 1 \\
\hline
\end{tabular}

\section{Appendix D. Fourgroup}

The symmetry properties of the rotational Hamiltonian, as well as rotational wavefunctions, of a rigid asymmetric top molecule may be deduced from its ellipsoid of inertia, which is symmetric not only to an identity operation $E$ but also to a rotation by $180^{\circ}$, a $C_{2}$ operation, about any of its principal axes of inertia. This set of symmetry operations forms the Fourgroup (Viergruppe), which is designated by $V(a, b, c)$ [17]. These symmetry operations cause the angular momentum to transform in the following manner [17]:

$$
\begin{aligned}
& E: \mathbf{J}_{a} \rightarrow \mathbf{J}_{a}, \mathbf{J}_{b} \rightarrow \mathbf{J}_{b}, \mathbf{J}_{c} \rightarrow \mathbf{J}_{c} \\
& C_{2}^{a}: \mathbf{J}_{a} \rightarrow \mathbf{J}_{a}, \mathbf{J}_{b} \rightarrow-\mathbf{J}_{b}, \mathbf{J}_{c} \rightarrow-\mathbf{J}_{c} \\
& C_{2}^{b}: \mathbf{J}_{a} \rightarrow-\mathbf{J}_{a}, \mathbf{J}_{b} \rightarrow \mathbf{J}_{b}, \mathbf{J}_{c} \rightarrow-\mathbf{J}_{c} \\
& C_{2}^{c}: \mathbf{J}_{a} \rightarrow-\mathbf{J}_{a}, \mathbf{J}_{b} \rightarrow-\mathbf{J}_{b}, \mathbf{J}_{c} \rightarrow \mathbf{J}_{c}
\end{aligned}
$$

The character table of the Fourgroup can is shown in Table D.2.

\section{Appendix E. Wang transformation}

The field-free semirigid rotor Hamiltonian operators $H_{\text {rigid }}+H_{\mathrm{d}}$ described above can be symmetrized to belong to the Fourgroup $\mathrm{V}$ and every field-free rotor wavefunction can be classified according to its behavior under $V(a, b, c)$ [17]. This symmetry classification is provided in Table E.3.

The symmetrized basis functions constructed by Wang transformation are defined as [17,23,42]:

$$
\begin{aligned}
& |J, 0, M, 0\rangle=|J, 0, M\rangle \text { for } K=0 \\
& |J, K, M, s\rangle=\frac{1}{\sqrt{2}}\left(|J, K, M\rangle+(-1)^{s}|J,-K, M\rangle\right) \text { for } K \neq 0
\end{aligned}
$$

where $s$ is 0 (symmetric) or 1 (antisymmetric) and $K$ now takes on only positive values. The Wang transformation can be expressed in a matrix form and the transformation matrix $X$ of order $(2 J+1)$ can be expressed as:

$$
X=X^{-1}=\tilde{X}=\frac{1}{\sqrt{2}}\left[\begin{array}{ccccccc}
\ddots & & & \vdots & & & . \\
& -1 & 0 & 0 & 0 & 1 & \\
& 0 & -1 & 0 & 1 & 0 & \\
\cdots & 0 & 0 & \sqrt{2} & 0 & 0 & \cdots \\
& 0 & 1 & 0 & 1 & 0 & \\
& 1 & 0 & 0 & 0 & 1 & \\
. & & & \vdots & & & \ddots
\end{array}\right] .
$$

The change of basis can be written as $\boldsymbol{\Psi}_{\text {Wang }}=\tilde{X} \boldsymbol{\Psi}$. For fixed values of $J$ and $M$, the vector $\boldsymbol{\Psi}$ consists of (2J +1$)$ symmetric top basis functions $|J, K, M\rangle$, whereas $\boldsymbol{\Psi}_{\text {Wang }}$ is the vector of new basis functions that contains the $(2 J+1)$ symmetrized functions $|J, K, M, s\rangle$ :

$$
\boldsymbol{\Psi}_{\text {Wang }}=\left(\begin{array}{c}
|J, J, M, 1\rangle \\
J,(J-1), M, 1\rangle \\
\vdots \\
J, 1, M, 1\rangle \\
|J, 0, M, 0\rangle \\
|J, 1, M, 0\rangle \\
\vdots \\
J,(J-1), M, 0\rangle \\
|J, J, M, 0\rangle
\end{array}\right), \quad \boldsymbol{\Psi}=\left(\begin{array}{c}
|J,-J, M\rangle \\
|J,(-J+1), M\rangle \\
\vdots \\
|J,-1, M\rangle \\
|J, 0, M\rangle \\
|J, 1, M\rangle \\
\vdots \\
|J,(J-1), M\rangle \\
|J, J, M\rangle
\end{array}\right) .
$$

In the new basis the Hamiltonian matrix factorizes into four sub-matrices that are historically denoted as $E^{+}, O^{+}, E^{-}, O^{-}[23,42]$ :

$$
H_{\text {Wang, rot }}=\tilde{X} H_{\text {rot }} X=E^{+}+O^{+}+E^{-}+O^{-}
$$

These sub-matrices are classified by the evenness and oddness of $K$ and $s$, as shown in Table E.3. For a single value of $J,|J, K, M, s\rangle$ wavefunctions within each sub-matrix all belong to the same symmetry species of $V[43,17]$ and the correlation is given in Table E.3. Thus, $H_{\text {Wang, rot }}$ can also be block-diagonalized in terms of four symmetry species, $A, B_{a}, B_{b}$, and $B_{c}$. This symmetrization of the basis by the Wang transformation simplifying the numerical evaluation and, most importantly, is necessary for the correct adiabatic labeling of the eigenstates in the electric field. 
Table E.3

Symmetry classification of asymmetric top wavefunctions $\left\langle J_{K_{a} K_{c}}, M\right\rangle$ for representation $I^{r}$ [18]. The symmetry species of each $J_{K_{a} K_{c}}$ is determined by the evenness or oddness of $K_{a}$ and $K_{c}$, which is indicated in parentheses in columns 4 and 5 . The classification of Wang sub-matrices is also provided.

\begin{tabular}{lllll}
\hline Sub-matrix & $K$ & $s$ & $J_{\text {even }}$ & $J_{\text {odd }}$ \\
\hline$E^{+}$ & $\mathrm{e}$ & 0 & $A(e e)$ & $B_{a}(e o)$ \\
$E^{-}$ & $\mathrm{e}$ & 1 & $B_{a}(e o)$ & $A(e e)$ \\
$O^{+}$ & 0 & 0 & $B_{b}(0 o)$ & $B_{c}(o e)$ \\
$O^{-}$ & 0 & 1 & $B_{c}(o e)$ & $B_{b}(o o)$ \\
\hline
\end{tabular}

Table F.4

Symmetries of asymmetric tops in dc electric fields. Different shapes represent the distinct symmetry species (matrix blocks) for the case of $M=0$ (open symbols) and $M \neq 0$ (filled symbols).

\begin{tabular}{|c|c|c|c|c|c|c|c|c|c|c|}
\hline \multirow[t]{3}{*}{$\mu_{a}$} & \multirow[t]{3}{*}{$\mu_{b}$} & \multirow[t]{3}{*}{$\mu_{c}$} & \multicolumn{4}{|l|}{ Jeven } & \multicolumn{4}{|l|}{ Jodd } \\
\hline & & & A & $B_{a}$ & $B_{b}$ & $B_{c}$ & A & $B_{a}$ & $B_{b}$ & $B_{c}$ \\
\hline & & & $E^{+}$ & $E^{-}$ & $\mathrm{O}^{+}$ & $O^{-}$ & $E^{-}$ & $E^{+}$ & $O^{-}$ & $\mathrm{O}^{+}$ \\
\hline$\neq 0$ & 0 & 0 & $\square$ & $\bigcirc$ & $\triangle$ & $\diamond$ & $\bigcirc$ & $\square$ & $\diamond$ & $\triangle$ \\
\hline 0 & $\neq 0$ & 0 & $\square$ & $\bigcirc$ & $\triangle \boldsymbol{\square}$ & $\diamond$ & $\triangle \boldsymbol{\square}$ & $\diamond$ & $\square$ & $\bigcirc$ \\
\hline 0 & 0 & $\neq 0$ & $\square$ & $\bigcirc$ & $\Delta$ & $\diamond \boldsymbol{\square}$ & $\diamond \boldsymbol{\square}$ & $\triangle$ & $\bigcirc$ & $\square \square$ \\
\hline$\neq 0$ & $\neq 0$ & 0 & $\square$ & $\diamond \mathbf{\square}$ & $\diamond \boldsymbol{\square}$ & $\square \square$ & $\diamond \mathbf{\square}$ & $\square$ & $\square \square$ & $\diamond \boldsymbol{\square}$ \\
\hline 0 & $\neq 0$ & $\neq 0$ & $\square$ & $\square \square$ & $\diamond \boldsymbol{\square}$ & $\diamond$ & $\diamond \boldsymbol{\square}$ & $\diamond \boldsymbol{\square}$ & $\square \square$ & $\square$ \\
\hline$\neq 0$ & 0 & $\neq 0$ & $\square \square$ & $\diamond \boldsymbol{\square}$ & $\square$ & $\diamond \mathbf{\square}$ & $\diamond \boldsymbol{\square}$ & $\square$ & $\diamond \mathbf{\square}$ & $\square \square$ \\
\hline$\neq 0$ & $\neq 0$ & $\neq 0$ & $\square$ & $\square$ & $\square \square$ & $\square \square$ & $\square$ & $\square$ & $\square \square$ & $\square \square$ \\
\hline
\end{tabular}

\section{Appendix F. Block diagonalization of the Hamiltonian matrix}

The Hamiltonian is block-diagonal in $M$ and the calculations are performed for each $M$ separately. In the field-free case all $M$ s are degenerate and only $M=0$ is calculated. For the symmetric top, $K$ is a good quantum number and the matrix is always also factorized into separate $K$ blocks. As mentioned in Appendix E, the Hamiltonian matrix of field-free symmetric or asymmetric tops can be block diagonalized into four blocks according to Fourgroup symmetry. An external dc electric field can mix these blocks, but the remaining symmetries allow partial factorization. In Table F.4 we summarize the block diagonalization of the Hamiltonian matrix in an electric field according to $V$ for all possible cases of non-zero dipole moment directions, i. e., all possible combinations of non-zero dipole-moment components in the principal axes of the inertial system. We note that the remaining symmetry can be higher for $M=0$ than for $M \neq 0$. This can also be seen from the matrix elements given above, where the $\Delta J=0$ Stark-coupling elements are always proportional to $M$, i. e., these couplings vanish for $M=0$.

The factorization summarized in Table F.4 can be understood in terms of the symmetry properties of the direction cosine $\phi_{Z_{g}}$ in (4) [17]. For the case of $\mu=\mu_{\alpha}$ and $M \neq 0$, basis functions of symmetries $A$ and $B_{\alpha}$ are coupled, as well as those of symmetries $B_{\alpha^{\prime}}$ and $B_{\alpha^{\prime \prime}}$, where $\alpha \neq \alpha^{\prime} \neq \alpha^{\prime \prime} \neq \alpha$. However, no coupling between these two subsets exists. The Hamiltonian matrix can thus be factorized into two blocks, as listed in Table F.4, one (filled square symbol) containing $A$ and $B_{\alpha}$ and the other one (filled diamond symbol) containing $B_{\alpha^{\prime}}$ and $B_{\alpha^{\prime \prime}}$. For the special case of $M=0$, states of symmetries $A$ and $B_{\alpha}$ for any given $J$ are also not coupled, nor are states of symmetries $B_{\alpha^{\prime}}$ and $B_{\alpha^{\prime \prime}}$ coupled [44]. This is due to the vanishing matrix elements (C.5), (C.7) and (C.9) for $M=0$. As a result, states of symmetry $A$ in $J_{\text {even }}\left(J_{\text {odd }}\right)$ only couple with those of symmetry $B_{\alpha}$ in $J_{\text {odd }}\left(J_{\text {even }}\right)$, etc. This remaining symmetry for the case $\mu=\mu_{\alpha}$ is represented by the open square symbol (open circle symbol) in the first line of Table F.4. States of symmetries $B_{\alpha^{\prime}}$ and $B_{\alpha^{\prime \prime}}$ also couple in the same manner, and their remaining symmetries are represented by open triangle and open diamond symbols in Table F.4. A similar behavior is observed for all cases when the dipole moment is along a principal axis of inertia. For the cases with more than one non-zero dipole moment component states of all four symmetry species $A, B_{a}, B_{b}$ and $B_{c}$ are coupled for $M \neq 0$ and only one symmetry species remains. For $M=0$ and one $\mu_{\alpha}=0$, the Hamiltonian matrix can be factorized into two blocks. For a dipole moment with components along all principal axes of inertia no partial Fourgroup symmetry remains in an electric field.

\section{References}

[1] S.Y.T. van de Meerakker, H.L. Bethlem, N. Vanhaecke, G. Meijer, Chem. Rev. 112 (2012) 4828-4878.

[2] F. Filsinger, G. Meijer, H. Stapelfeldt, H.N. Chapman, J. Küpper, Phys. Chem. Chem. Phys. 13 (2011) 2076-2087.

[3] M. Schnell, G. Meijer, Angew. Chem. Int. Ed. Engl. 48 (2009) 6010-6031.

[4] M.T. Bell, T.P. Softley, Mol. Phys. 107 (2009) 99-132.

[5] J. Küpper, F. Filsinger, G. Meijer, Faraday Discuss. Chem. Soc. 142 (2009) 155-173.

[6] L. Holmegaard, J.H. Nielsen, I. Nevo, H. Stapelfeldt, F. Filsinger, J. Küpper, G. Meijer, Phys. Rev. Lett. 102 (2009) 023001.

[7] F. Filsinger, U. Erlekam, G. von Helden, J. Küpper, G. Meijer, Phys. Rev. Lett. 100 (2008) 133003.

[8] K. Wohlfart, F. Grätz, F. Filsinger, H. Haak, G. Meijer, J. Küpper, Phys. Rev. A 77 (2008) 031404(R).

[9] J.H. Nielsen, P. Simesen, C.Z. Bisgaard, H. Stapelfeldt, F. Filsinger, B. Friedrich, G. Meijer, J. Küpper, Phys. Chem. Chem. Phys. 13 (2011) $18971-18975$.

[10] F. Filsinger, J. Küpper, G. Meijer, J.L. Hansen, J. Maurer, J.H. Nielsen, L. Holmegaard, H. Stapelfeldt, Angew. Chem. Int. Ed. Engl. 48 (2009) $6900-6902$.

[11] S. Trippel, Y.-P. Chang, S. Stern, T. Mullins, L. Holmegaard, J. Küpper, Phys. Rev. A 86 (2012) 033202.

[12] B.C. Dian, G.G. Brown, K.O. Douglass, B.H. Pate, Science 320 (2008) 924-928.

[13] F. Hennies, A. Pietzsch, M. Berglund, A. Föhlisch, T. Schmitt, V. Strocov, H.O. Karlsson, J. Andersson, J.-E. Rubensson, Phys. Rev. Lett. 104 (2010) 193002.

[14] G. Sciaini, R.J.D. Miller, Rep. Progr. Phys. 74 (2011) 096101.

[15] A. Barty, J. Küpper, H.N. Chapman, Annu. Rev. Phys. Chem. 64 (2013) 415-435.

[16] J. Küpper, et al. 2013. arXiv:1307.4577 [physics].

[17] W. Gordy, R.L. Cook, Microwave Molecular Spectra, third ed., John Wiley \& Sons, New York, NY, USA, 1984

[18] R.N. Zare, Angular Momentum, John Wiley \& Sons, New York, NY, USA, 1988

[19] M. Okruss, R. Müller, A. Hese, J. Chem. Phys. 110 (1999) 10393-10402. 
[20] F. Filsinger, J. Küpper, G. Meijer, L. Holmegaard, J.H. Nielsen, I. Nevo, J.L. Hansen, H. Stapelfeldt, J. Chem. Phys. 131 (2009) 064309.

[21] J.K.G. Watson, Can. J. Phys 53 (1975) 2210-2220.

[22] P.R. Bunker, P. Jensen, Molecular Symmetry and Spectroscopy, second ed., NRC Research Press, Ottawa, Ontario, Canada, 1998.

[23] S.C. Wang, Phys. Rev. 34 (1929) 243-252.

[24] H.L. Bethlem, M.R. Tarbutt, J. Küpper, D. Carty, K. Wohlfart, E.A. Hinds, G. Meijer, J. Phys. B 39 (2006) R263-R291.

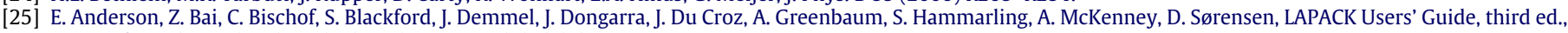
Society for Industrial and Applied Mathematics, Philadelphia, PA, USA, 1999.

[26] F. Filsinger, K. Wohlfart, M. Schnell, J.-U. Grabow, J. Küpper, Phys. Chem. Chem. Phys. 10 (2008) 666-673.

[27] C.M. Western, PGOPHER, A Program for Simulating Rotational Structure, University of Bristol, Bristol, UK, 2013, URL: http://pgopher.chm.bris.ac.uk.

[28] H.M. Pickett, J. Mol. Spectrosc. 148 (1991) 371-377.

[29] H.M. Pickett, SPFIT/SPCAT, programs for fitting and predictions in rotational spectroscopy, 1991-2007. URL: http://spec.jpl.nasa.gov.

[30] Z. Kisiel, B.A. Pietrewicz, P.W. Fowler, A.C. Legon, E. Steiner, J. Phys. Chem. A 104 (2000) 6970-6978.

[31] Z. Kisiel, J. Kosarzewski, B.A. Pietrewicz, L. Pszczółkowski, Chem. Phys. Lett. 325 (2000) 523-530.

[32] Z. Kisiel, qstark website. URL: http://www.ifpan.edu.pl/ kisiel/dip/dip.htm\#qstark (accessed 2013).

[33] D.R. Borst, Ph.D. Thesis, University of Pittsburgh, Department of Chemistry, Pittsburgh, PA, USA, 2001.

[34] C. Kang, T.M. Korter, D.W. Pratt, J. Chem. Phys. 122 (2005) 174301.

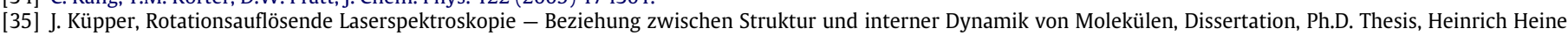
University, Faculty of Mathematics and Sciences, Düsseldorf, Germany, 2000.

[36] F.W. Birss, D.A. Ramsay, Comput. Phys. Commun. 38 (1984) 83-112.

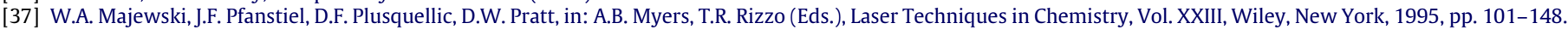

[38] W.L. Meerts, M. Schmitt, G.C. Groenenboom, Can. J. Chem. 82 (2004) 804-819.

[39] F. Filsinger, S. Putzke, H. Haak, G. Meijer, J. Küpper, Phys. Rev. A 82 (2010) 052513.

[40] J.K.G. Watson, in: J.R. Durig (Ed.), Vibrational Spectra and Structure, Vol. 6, Marcel Dekker, 1977, p. 1.

[41] P.C. Cross, R.M. Hainer, G.W. King, J. Chem. Phys. 12 (1944) 210-243.

[42] R. Mulliken, Phys. Rev. 59 (1941) 873-889.

[43] G.W. King, R.M. Hainer, P.C. Cross, J. Chem. Phys. 11 (1943) 27-42.

[44] R. Escribano, B. Mate, F. Ortigoso, J. Ortigoso, Phys. Rev. A 62 (2000) 023407. 